University of Wollongong

Research Online

Faculty of Engineering and Information

Faculty of Engineering and Information

Sciences - Papers: Part A

Sciences

$1-1-2012$

\title{
Expectation of trading agent behaviour in negotiation of electronic
} marketplace

Fenghui Ren

University of Wollongong, fren@uow.edu.au

Minjie Zhang

University of Wollongong, minjie@uow.edu.au

John Fulcher

University of Wollongong, john@uow.edu.au

Follow this and additional works at: https://ro.uow.edu.au/eispapers

Research Online is the open access institutional repository for the University of Wollongong. For further information contact the UOW Library: research-pubs@uow.edu.au 


\title{
Expectation of trading agent behaviour in negotiation of electronic marketplace
}

\begin{abstract}
Electronic Commerce has been a very significant commercial phenomenon in recent years, and autonomous agents are widely adopted by business or individuals in electronic marketplaces to fulfill time consuming tasks in trading. Agent negotiation mechanisms are usually applied between conflicted agents in order to reach a mutually beneficial agreement. Prediction of trading agents' strategies and behaviours in negotiation is a very significant research topic in agent negotiation. By employing the prediction results on opponents' possible strategies and behaviours during a negotiation, trading agents can plan and perform corresponding strategies in order to maximize their own profits. Significant achievements have been made on this topic. However, most existing approaches are based on machine learning mechanisms, which may fail to capture opponents' behaviours in open and dynamic electronic marketplaces. In this paper, two agent behaviour expectation approaches are introduced to help trading agents to capture opponents' potential behaviours during a negotiation in complex e-marketplaces. (i) The regression analysis approach focuses on illustrating the main trends of opponents' trading behaviours; (ii) the vector analysis approach pays more attention to identifying opponents' detailed negotiation strategies. The experimental results show the efficiency and efficacy of the two proposed approaches in open and dynamic negotiation environments.
\end{abstract}

\section{Keywords}

trading, electronic, negotiation, expectation, marketplace, behaviour, agent

\section{Publication Details}

Ren, F., Zhang, M. \& Fulcher, J. (2012). Expectation of trading agent behaviour in negotiation of electronic marketplace. Web Intelligence and Agent Systems: an international journal, 10 (1), 49-63. 


\title{
Expectation of trading agent behaviour in negotiation of electronic marketplace
}

\author{
Fenghui Ren *, Minjie Zhang and John Fulcher \\ School of Computer Science and Software Engineering, University of Wollongong, Australia \\ E-mail: \{fren,minjie,john\}@uow.edu.au
}

\begin{abstract}
Electronic Commerce has been a very significant commercial phenomenon in recent years, and autonomous agents are widely adopted by business or individuals in electronic marketplaces to fulfill time consuming tasks in trading. Agent negotiation mechanisms are usually applied between conflicted agents in order to reach a mutually beneficial agreement. Prediction of trading agents' strategies and behaviours in negotiation is a very significant research topic in agent negotiation. By employing the prediction results on opponents' possible strategies and behaviours during a negotiation, trading agents can plan and perform corresponding strategies in order to maximize their own profits. Significant achievements have been made on this topic. However, most existing approaches are based on machine learning mechanisms, which may fail to capture opponents' behaviours in open and dynamic electronic marketplaces. In this paper, two agent behaviour expectation approaches are introduced to help trading agents to capture opponents' potential behaviours during a negotiation in complex e-marketplaces. (i) The regression analysis approach focuses on illustrating the main trends of opponents' trading behaviours; (ii) the vector analysis approach pays more attention to identifying opponents' detailed negotiation strategies. The experimental results show the efficiency and efficacy of the two proposed approaches in open and dynamic negotiation environments.
\end{abstract}

Keywords: Agent negotiation, e-marketplace, agent behaviour prediction

\section{Introduction}

Electronic Commerce (e-commerce) has been changing traditional methods of business in recent years and has become a very important commercial phenomenon $[11,14]$. Nowadays, many businesses operate in electronic marketplaces (e-markets). By comparison with traditional marketplaces, e-markets have some obvious advantages. (1) The e-market provides wider trading environments to participators than the traditional marketplace. (2) The e-market saves participators' costs on physical resources compared with traditional marketplaces. And (3) the e-market provides efficient instruments for communicating and trading between participators. For example, in e-marketplaces, merchants can save their budgets on business maintenance by avoiding physical shops and shop assistants. Also, shoppers do not need to visit shops in person which can save

\footnotetext{
*Corresponding author.
}

costs on traffic and time. Moreover, all participators can collect information about their items of interest and communicate with potential trading partners in a timely manner. Participators in an e-market are usually busy in collecting information, selecting trading partners, comparing similar goods and bargaining with different trading partners. In order to release participators from these time consuming jobs, autonomous agents are employed on behalf of traders to fulfil trading activities in e-markets. Participators only need 'tell' agents about their trading expectations, and then agents can perform the detailed dealing procedures automatically. However, because agents may have different trading purposes and strategies, conflicts may happen when they perform detailed trading behaviours in an e-market. Negotiation mechanisms are usually applied between conflicted agents in order to reach a mutually beneficial agreement among them $[21,27,29]$. In Fig. 1, we display an example of agent negotiation. Six agents (three buyers and three sellers) bargain over is- 


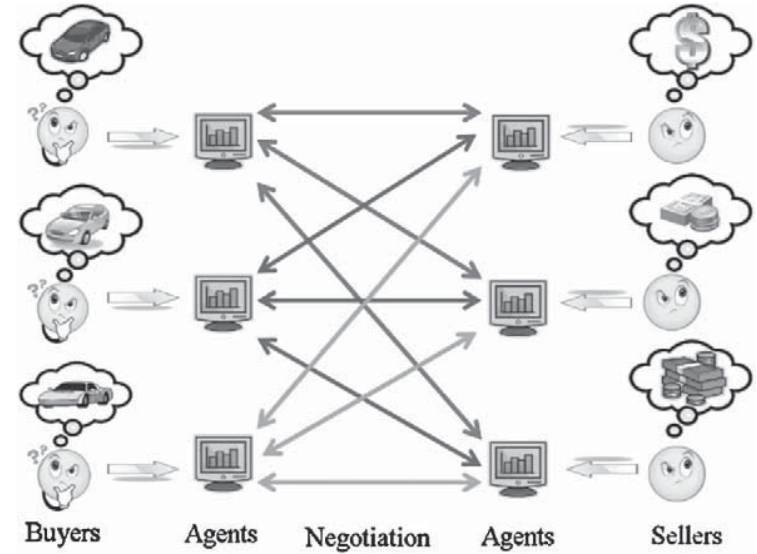

Fig. 1. A 3-to-3 multilateral negotiation.

sues related to purchasing a car. The buyers have different preferences on car models, colors and accessories, and the sellers concentrate more on profiles they can get. In order to reach an agreement by considering individual expectations, agent negotiation is adopted in such a situation. The detailed concepts about agent negotiation are introduced in the following paragraph.

Negotiation is a means for agents to communicate and compromise to reach mutually beneficial agreements $[7,16]$. However, in most situations, agents do not have complete information about their partners' negotiation strategies, and may have difficulty in making decisions on future negotiations, such as how to select suitable partners for further negotiation $[1,18]$, or how to generate a suitable counter-offer in the next negotiation round [20]. Therefore estimation approaches which can predict uncertain situations and possible changes in the future are required to help agents generate good and efficient negotiation strategies. Research on partner behaviour estimation has been a very active direction in recent years. By employing the expectation results on opponents' possible strategies and behaviours, self-interested agents can plan and perform corresponding behaviours during a negotiation in order to maximize their own profits. The literature includes significant achievements on this topic [3-5,12, $13,19,23,30,31]$. However, most existing approaches are based on machine learning mechanisms, i.e. the behaviour estimation approaches are well trained by samples in advance. Such mechanisms may perform well when the negotiation environment and opponents' behaviours are simple, i.e. the negotiation environment does not change during the negotiation and opponents do not modify their negotiation strategies frequently. However, in a real e-market, participators can enter into or leave off a marketplace freely. Hence, a negotiation environment may become highly dynamic, and opponents may modify their negotiation strategies frequently in order to maximize their profits. Therefore, when the machine learning based approach is used in real-world applications, some limitations will emerge.

In general, a machine learning based approach comprises two steps in order to properly estimate the agents' behaviour. In the first step, the proposed estimation function is required to be well trained by training data. Therefore, the performance of the estimation function is somehow decided by the training result. The training data could be both synthetic or collected from the real world. Usually, the synthetic data are helpful in training a function to enhance its problem solving skill for some particular issues, while the real world data can help the function to improve its ability in complex problem solving. After the estimation function is trained, it is employed to predict opponents' behaviours in the second step. However, no matter how many data are employed to train the estimation function, the training data may still not be comprehensive enough to cover all situations in reality. Therefore, it is very likely that the behaviour estimation results cannot truly reflect an opponent's behaviour which is not included in the training data. Also, when the negotiation environment becomes more open and dynamic, agents with different purposes, preferences and negotiation strategies can enter into and leave a negotiation dynamically. The machine learning based behaviours estimation approaches may not work well in such an uncertain situation by considering the limitations of (1) lacking sufficient data to train the estimation function, and (2) requesting extra trading time.

In this paper, two approaches for agent behaviour estimation are introduced to solve agent behaviour estimation problems in open and dynamic environments from different perspectives. The first approach is based on a regression analysis mechanism. By dynamically analyzing historical offers from an opponent, a power regression function is generated to fit the opponent's behaviour in each negotiation round dynamically and optimally. Then by analyzing the regression function, the opponent's behaviours can be identified. The estimation on the opponent's possible behaviour in the following negotiation round is indicated by a confidence bound. By comparing the difference between the opponent's behaviour in the historical record and the estimation results, the accuracy level of the regression function can also be calculated. Based on an agent's requirements on the accuracy of estimation results, the 
form of the regression function can also be changed dynamically during the negotiation. Such a regression estimation approach is very suitable for handling the opponent behaviour prediction problem in uncertain environments, and to identify the main trends of an opponent's trading behaviour.

The second approach pays more attention to detailed trends of an opponent's behaviour. According to the extent of the opponent's concessions during a negotiation, the opponent's possible behaviours are preclassified into several classes. During a negotiation, because an opponent may apply different strategies to make concessions in different periods of the negotiation, a vector analysis method is introduced to identify the opponent's concession strategies in each negotiation period. Then, based on the opponent's historical concession strategies, the possible concession strategy in the following negotiation round can be estimated. Compared with the regression approach, this vector approach will give more detailed information about an opponent's behaviour in different negotiation periods. Agents can get more information about an opponent's preferred negotiation strategies and behaviours in different negotiation periods and situations.

By comparison with machine learning mechanisms, the proposed two approaches only use the historical offers in the current negotiation to estimate an opponent's behaviour in future negotiations and do not require any additional training process. So the proposed approaches are very suitable to work under an open and dynamic negotiation environment, and to make timely credible judgements on an opponent's behaviour. Also, because the proposed approaches do not make any strict assumption on an agent's purpose, preference and negotiation strategy, they can be employed widely in negotiation by different types of agents. Furthermore, the proposed regression approaches not only represent the estimation results within bound, but also give the probability that each individual situation may happen in future. Thus an agent can easily have an overview on an opponent's possible behaviours, and then modify its own negotiation strategy based on this information.

The rest of the paper is organized as follows. In Section 2, we introduce the power regression approach to estimate an opponent's negotiation behaviour. In Section 3, we introduce the vector analysis approach to show how to predict an opponent's behaviour. Section 4 introduces an approach to calculate the probability that predicted behaviours may happen in the future. Section 5 discusses the experimental results of the two proposed estimation approaches, and also a com- parison between related approaches. In Section 6, related work on agent behaviour estimation is introduced and compared with the proposed approaches. Section 7 concludes this paper and suggests future work.

\section{Regression analysis}

In this section, we introduce a power regression approach to analyse an opponent's historical offers and to predict the opponent's possible negotiation behaviours in the future.

\subsection{Background}

In this subsection, we introduce the background about the proposed power regression function for opponent behaviour estimation in negotiation. The regression analysis employ both mathematics and probability theory, and can estimate the strength of a modeled relationship between one or more dependent and independent variables. In order to simplify the complexity for the proposed regression analysis approach, we make the following simple assumption about a negotiation:

The utilities that an agent gains from an opponent in previous negotiation rounds is a sequence in monotonic ascending order.

The reason behind this assumption is based on the consideration that a negotiator cannot break its previous promises during a negotiation. Generally, there are four kinds of behaviour which an agent can perform in a negotiation, namely Boulware, Linear, Conceder and Sit-and-Wait $[6,8,28]$.

In Fig. 2, we illustrate these four common agent behaviours. Let the $\mathrm{x}$-axis indicate the negotiation round and the $y$-axis represents the concession that an agent can make in a negotiation. Details of the four common negotiation strategies are as follows:

- Boulware: the rate of change in the slope is increasing, corresponding to smaller concessions in the early stage of a negotiation, but large concessions in the later stage of the negotiation.

- Linear: the rate of change in the slope is zero, corresponding to a constant concession throughout a negotiation.

- Conceder: the rate of change in the slope is decreasing, corresponding to large concessions in the early stage of a negotiation, but smaller concessions in the later stage of the negotiation. 


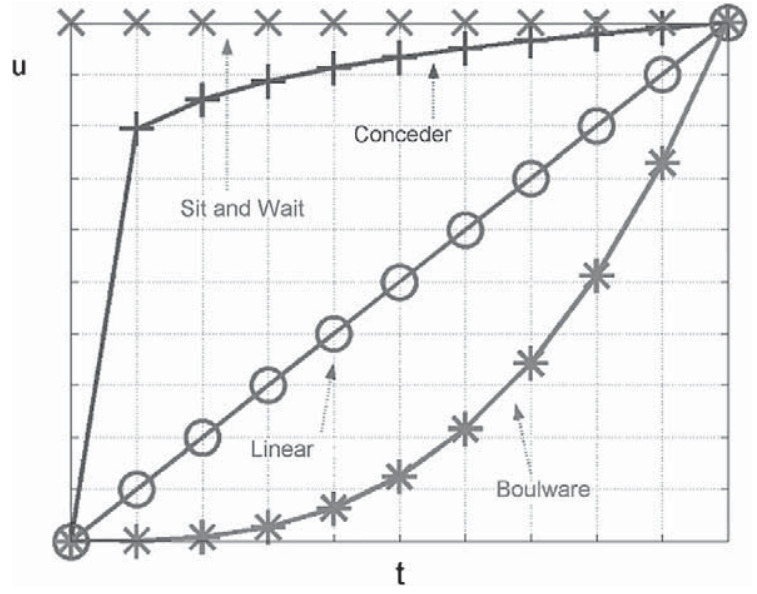

Fig. 2. Four common strategies in agent negotiation.

- Sit-and-Wait: the rate of change of the slope and the slope itself are always zero, corresponding to not making any concession throughout a negotiation.

Since the curves of an agent's possible behaviours illustrated in Fig. 2 are monotonic, we introduce a power regression function to predict an agent's behaviour in single-issue bilateral negotiation as follows:

$$
U(t)=a \times t^{b}
$$

where $U(t)$ is the utility gained from an opponent at round $t(0 \leqslant t \leqslant \tau, \tau$ is the agent negotiation deadline), and both $a$ and $b(a \geqslant 0, b \geqslant 0)$ are independent of $t$. It is noticed that the four negotiation strategies can be represented by Eq. (1) with different $b$ values:

- Boulware: when $b>1$, the rate of change in the slope is decreasing, corresponding to smaller concessions in the early stage but large concession in later cycles.

- Linear: when $b=1$, the rate of change in the slope is zero, corresponding to a constant concession throughout a negotiation.

- Conceder: when $0<b<1$, the rate of change in the slope is increasing, corresponding to large concessions in the early stage but smaller concession in the later stage.

- Sit-and-Wait: when $b=0$, the rate of change of the slope and the slope itself are always zero, corresponding to not making any concession throughout a negotiation.

In the following subsection, we will introduce the proposed power regression function to analyze and estimate possible opponent behaviours.

\subsection{Regression analysis on partners' behaviours}

In this subsection, we introduce a power regression analysis approach on a negotiation between two negotiators. Firstly, we perform the following equivalence transformation on Eq. (1):

$$
\begin{aligned}
\ln (U(t)) & =\ln \left(a \times t^{b}\right) \\
& =\ln (a)+b \times \ln (t)
\end{aligned}
$$

Then let $U(t)^{*}=\ln (U(t)), a^{*}=\ln (a)$ and $t^{*}=$ $\ln (t)$, Eq. (2) can be rewritten as:

$$
U(t)^{*}=a^{*}+b \times t^{*}
$$

The new function indicates a linear relationship between the variables $t^{*}$ and $U(t)^{*}$. Both coefficients $a^{*}$ and $b$ are independent of $t^{*}$. Let $\hat{u}_{t}$ be the real utility that an agent gained from an opponent at round $t$. The difference between the agent's real gained utility $\left(\hat{u}_{t}\right)$ and the expected utility $U(t)^{*}$ can be expressed by the formula $\varepsilon(t)=\hat{u}_{t}-a^{*}+b \times t^{*}$. It is assumed that the distribution of $\varepsilon(t)$ obeys the Gaussian distribution, i.e. $\varepsilon \sim N\left(0, \sigma^{2}\right)$.

Let pairs $\left\{\left(t_{i}, \hat{u}_{i}\right)\right\}(i \in[0, n])$ be the historical records of an agent's utilities in the previous $n$ rounds in the current negotiation, where $t_{i}\left(t_{i}<t_{i+1}\right)$ indicates the $i^{\text {th }}$ negotiation round, and $\hat{u}_{i}\left(\hat{u}_{i} \leqslant \hat{u}_{i+1}\right)$ indicates the real utility that the agent gained from the opponent. Firstly, we transform all pairs of $\left(t_{i}, \hat{u}_{i}\right)$ to $\left(t_{i}^{*}, \hat{u}_{i}^{*}\right)$ as follows.

$$
\left\{\begin{array}{l}
t_{i}^{*}=\ln \left(t_{i}\right) \\
\hat{u}_{i}^{*}=\ln \left(u_{i}\right)
\end{array}\right.
$$

Because $\hat{u}_{i}^{*}=U(t)^{*}+\varepsilon_{i}$, where $\varepsilon_{i}$ is the estimation error in round $i$ and the distribution of all $\varepsilon_{i}$ obeys $N\left(0, \sigma^{2}\right)$, so the joint probability density function for $U(t)^{*}$ is:

$$
\begin{aligned}
L & =\prod_{i=1}^{n} \frac{1}{\sigma \sqrt{2 \pi}} \exp \left[-\frac{1}{2 \sigma^{2}}\left(\hat{u}_{i}^{*}-U(t)^{*}\right)^{2}\right] \\
& =\prod_{i=1}^{n} \frac{1}{\sigma \sqrt{2 \pi}} \exp \left[-\frac{1}{2 \sigma^{2}}\left(\hat{u}_{i}^{*}-a^{*}-b t_{i}^{*}\right)^{2}\right] \\
& =\left(\frac{1}{\sigma \sqrt{2 \pi}}\right)^{n} \exp \left[-\frac{1}{2 \sigma^{2}} \sum_{i=1}^{n}\left(\hat{u}_{i}^{*}-a^{*}-b t_{i}^{*}\right)^{2}\right]
\end{aligned}
$$

Because the purpose of this regression approach is to find a function $U(t)^{*}$ to fit all real utilities 
optimally, so the joint probability density function for $U(t)^{*}$ should be maximized. In order to make $L$ achieve its maximum, obviously $\sum_{i=1}^{n}\left(\hat{u}_{i}^{*}-a^{*}-b t_{i}^{*}\right)^{2}$ should achieve its minimum value. Let $Q\left(a^{*}, b\right)=$ $\sum_{i=1}^{n}\left(\hat{u}_{i}^{*}-a^{*}-b t_{i}^{*}\right)^{2}$, we calculate the first-order partial derivative for $Q\left(a^{*}, b\right)$ on both $a^{*}$ and $b$, and let the results equal zero, which are shown as follows:

$$
\left\{\begin{array}{l}
\frac{\partial Q}{\partial a^{*}}=-2 \sum_{i=1}^{n}\left(\hat{u}_{i}^{*}-a^{*}-b t_{i}^{*}\right)=0 \\
\frac{\partial Q}{\partial b}=-2 \sum_{i=1}^{n}\left(\hat{u}_{i}^{*}-a^{*}-b t_{i}^{*}\right) t_{i}^{*}=0
\end{array}\right.
$$

then,

$$
\left\{\begin{array}{l}
n a^{*}+\left(\sum_{i=1}^{n} t_{i}^{*}\right) b=\sum_{i=1}^{n} \hat{u}_{i}^{*} \\
\left(\sum_{i=1}^{n} t_{i}^{*}\right) a^{*}+\left(\sum_{i=1}^{n} t_{i}^{* 2}\right) b=\sum_{i=1}^{n} t_{i}^{*} \hat{u}_{i}^{*}
\end{array}\right.
$$

Because Eq. (7)'s coefficient matrix is:

$$
\begin{aligned}
\left|\begin{array}{cc}
n & \sum_{i=1}^{n} t_{i}^{*} \\
\sum_{i=1}^{n} t_{i}^{*} & \sum_{i=1}^{n} t_{i}^{* 2}
\end{array}\right| & =n \sum_{i=1}^{n} t_{i}^{* 2}-\left(\sum_{i=1}^{n} t_{i}^{*}\right)^{2} \\
& =n \sum_{i=1}^{n}\left(t_{i}^{*}-\bar{t}\right)^{2} \neq 0
\end{aligned}
$$

So coefficients $a^{*}$ and $b$ have a unique solution as follows:

$$
\left\{\begin{array}{l}
b=\frac{n \sum_{i=1}^{n} t_{i}^{*} \hat{u}_{i}^{*}-\left(\sum_{i=1}^{n} t_{i}^{*}\right)\left(\sum_{i=1}^{n} u_{i}^{*}\right)}{n \sum_{i=1}^{n} t_{i}^{* 2}-\left(\sum_{i=1}^{n} t_{i}^{*}\right)^{2}} \\
a^{*}=\frac{1}{n} \sum_{i=1}^{n} \hat{u}_{i}^{*}-\frac{b}{n} \sum_{i=1}^{n} t_{i}^{*}
\end{array}\right.
$$

In order to simplify the solution, let

$$
\left\{\begin{array}{l}
S_{x x}=\sum_{i=1}^{n} t_{i}^{* 2}-\frac{1}{n}\left(\sum_{i=1}^{n} t_{i}^{*}\right)^{2} \\
S_{x y}=\sum_{i=1}^{n} t_{i}^{*} \hat{u}_{i}^{*}-\frac{1}{n}\left(\sum_{i=1}^{n} t_{i}^{*}\right)\left(\sum_{i=1}^{n} \hat{u}_{i}^{*}\right)
\end{array}\right.
$$

then $a^{*}$ and $b$ are represented as:

$$
\left\{\begin{array}{l}
b=\frac{S_{x y}}{S_{x x}} \\
a^{*}=\frac{1}{n} \sum_{i=1}^{n} \hat{u}_{i}^{*}-\left(\frac{1}{n} \sum_{i=1}^{n} t_{i}^{*}\right) b
\end{array}\right.
$$

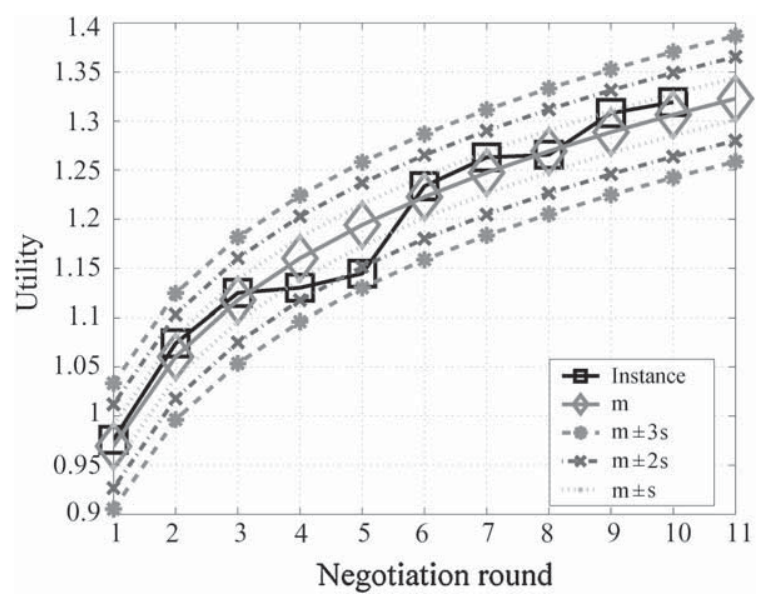

Fig. 3. Estimation of agent's negotiation behaviour by using regression analysis.

and finally let $a=\exp \left(a^{*}\right)$, then the prediction function is:

$$
U(t)=\exp \left(a^{*}\right) \times t^{b}
$$

In Fig. 3, we illustrate an example of the regression analysis. A power regression curve is generated to optimally fit all historical offers from an opponent. Also, the confidence area of the regression curve is displayed between $\pm 3 \sigma$ (see Section 4 for details).

\section{Vector analysis}

In this section, we introduce a vector analysis approach to predict an opponent's behaviour during a negotiation. The prediction is still only based on historical offers of the current negotiation. The vector analysis approach has three major steps. Firstly, the historical records of previous offers are classified according to the difference between each pair of historical offers. Secondly, offers in the same class are regressed by adopting a linear function. Thirdly, results of different linear regression functions are combined to generate the final prediction result. The detailed procedure of the three steps is introduced in the following subsections.

\subsection{Classification}

In this subsection, we introduce a vector classification approach to classify historical offers according to the difference between each two historical offers. Let pairs $\left\{\left(t_{i}, \hat{u}_{i}\right)\right\}(i \in[0, n])$ be the historical records 


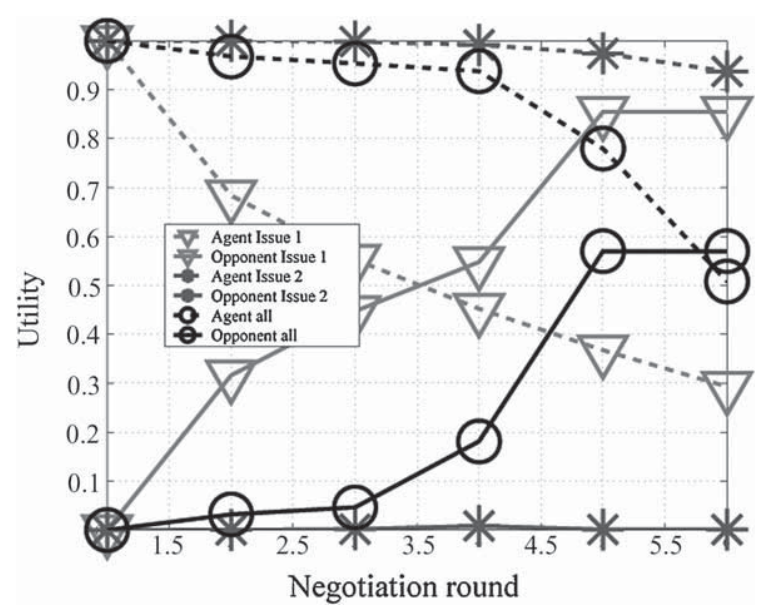

Fig. 4. An example of two-issue negotiation.

of an opponent's offers in the previous $n$ rounds in the current negotiation, where $t_{i}\left(t_{i}<t_{i+1}\right)$ indicates the $i^{\text {th }}$ negotiation round and $\hat{u}_{i}\left(\hat{u}_{i} \in[0,1]\right)$ indicates the real utility that the agent gained from the opponent during round $t_{i}$. One possible case of two agent twoissue negotiation is illustrated in Fig. 4, where the $x$ axis indicates the negotiation round, and the $y$-axis indicates the utility. The lines marked by triangles is the negotiation on Issue 1 , the lines marked by asterisks is the negotiation on Issue 2 , and the lines marked by circles is the negotiation by considering both issues. Each point in the graph indicates an offer. The graph shows all offers from two agents in the first six rounds. It can be seen that agents may have different strategies to make concessions for different issues and/or in different periods of a negotiation. So according to how fast an agent gives a concession - the slope of the line between two points - the agent's historical offers can be classified.

The basic idea of the vector clustering is to classify offers according to the slope of the line between each pairwise in the data collection. So, the offers in the same cluster will have a similar concession rate. Firstly, degrees of lines between one offer and all other offers is calculated. The dominant degree will be the cluster which this offer should belong to. By repeating this process to all historical offers, each offer can be classified into a particular cluster. The detailed procedure of the vector classification is listed in Algorithm 1.

An example of the vector classification is displayed in Fig. 5. In this example, 11 points are going to be classified into a maximum of 18 classes $\left(10^{\circ}\right.$ for each class). Taking point $p 1$ as an instance, by calculating the slope of lines between the point $p 1$ and other

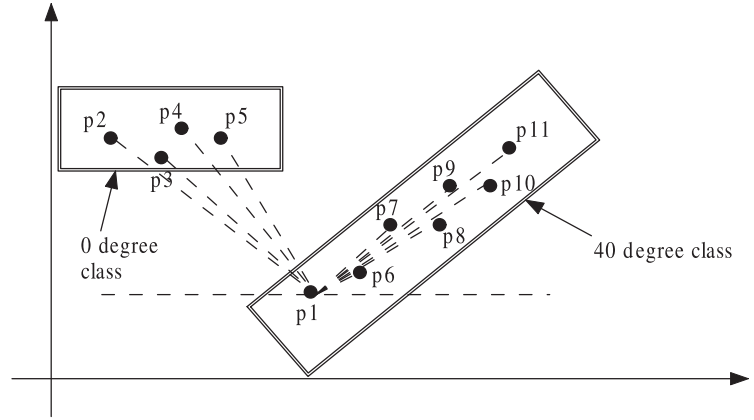

Fig. 5. Classify point $p 1$ by using vector classification.

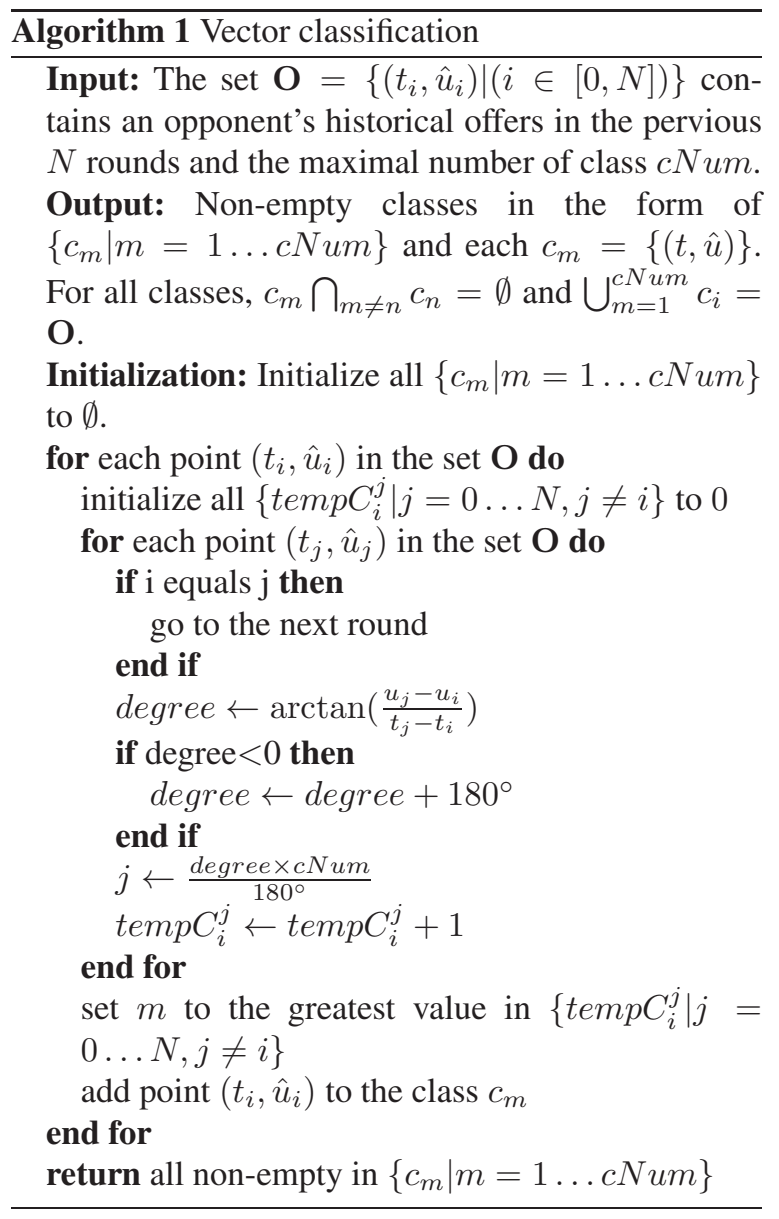

points, it is found that the degrees of angles between the point $p 1$ and points $p 6, p 7, p 8, p 9, p 10$, and $p 11$ are in the range $\left[35^{\circ}, 45^{\circ}\right]$. The degrees of angles between the point $p 1$ and points $p 2$ and $p 3$ are in the range $\left[135^{\circ}, 145^{\circ}\right]$. The degrees of angles between the point $p 1$ and points $p 4$ and $p 5$ are in the range $\left[125^{\circ}, 135^{\circ}\right]$. Since the dominating angles between $p 1$ and other points are in the range $\left[35^{\circ}, 45^{\circ}\right]$. So point $p 1$ is clas- 
sified as the $40^{\circ}$ class. By repeating such a process, all points are finally classified into two classes as shown in Fig. 5 , i.e. points $p 2, p 3, p 4$ and $p 5$ belong to $0^{\circ}$ class, and other points belong to $40^{\circ}$ class.

Because offers in the same cluster will distribute along a straight line, then in the next section, a linear regression approach will be employed to identify patterns in each cluster with one or more linear regression functions.

\subsection{Multiple linear regression}

In the previous subsection, we introduced a vector classification approach to classify historical offers in negotiation according to their slopes, i.e., how much concession an agent would like to make at a certain moment of a negotiation. After the classification, historical offers in the same class have similar slopes and we can simply adopt a linear regression approach to analyzing each class. Now replacing $U(t)^{*}$ by $U(t)=$ $b \times t+a, \hat{u}_{i}^{*}$ by $\hat{u}_{i}$, and $t_{i}^{*}$ by $t_{i}$ in Eq. (5), we can easily find the parameters $a$ and $b$ to optimally fit all offers in the same class by the function $U(t)=b \times t+a$. The two coefficients $a$ and $b$ are calculated as follows.

$$
\left\{\begin{array}{l}
a=\frac{\mathbf{M}_{\mathbf{a}}}{\mathbf{M}} \\
b=\frac{\mathbf{M}_{\mathbf{b}}}{\mathbf{M}}
\end{array}\right.
$$

where

$$
\begin{aligned}
\mathbf{M}_{\mathbf{a}} & =\left|\begin{array}{cc}
\sum_{i=1}^{n}\left(t_{i} \cdot \hat{u}_{i}^{*}\right) & \sum_{i=1}^{n} t_{i} \\
\sum_{i=1}^{n} \hat{u}_{i}^{*} & n
\end{array}\right| \\
& =n \cdot \sum_{i=1}^{n}\left(t_{i} \cdot \hat{u}_{i}^{*}\right)-\sum_{i=1}^{n} t_{i} \cdot \sum_{i=1}^{n} \hat{u}_{i}^{*}, \\
\mathbf{M}_{\mathbf{b}} & =\left|\begin{array}{cc}
\sum_{i=1}^{n} t_{i}^{2} & \sum_{i=1}^{n}\left(t_{i} \cdot \hat{u}_{i}^{*}\right) \\
\sum_{i=1}^{n} t_{i} & \sum_{i=1}^{n} \hat{u}_{i}^{*}
\end{array}\right| \\
& =\sum_{i=1}^{n} \hat{u}_{i}^{*} \cdot \sum_{i=1}^{n} t_{i}^{2}-\sum_{i=1}^{n} t_{i} \cdot \sum_{i=1}^{n}\left(t_{i} \cdot \hat{u}_{i}^{*}\right)
\end{aligned}
$$

and

$$
\begin{aligned}
\mathbf{M} & =\left|\begin{array}{cc}
\sum_{i=1}^{n} t_{i}^{2} & \sum_{i=1}^{n} t_{i} \\
\sum_{i=1}^{n} t_{i} & n
\end{array}\right| \\
& =n \cdot \sum_{i=1}^{n} t_{i}^{2}-\left(\sum_{i=1}^{n} t_{i}\right)^{2}
\end{aligned}
$$

$$
\begin{aligned}
& =n \sum_{i=1}^{n}\left(t_{i}-\overline{t_{i}}\right)^{2} \\
& \neq 0
\end{aligned}
$$

where $\bar{t}_{i}$ is the average of all $t_{i}$, and the detailed procedure to calculate the parameters $a$ and $b$ can be found in Section 2.

By applying coefficients $a$ and $b$, the pattern in cluster $c_{i}$ can be represented. Even though the estimated regression function $U(t)$ can guarantee minimal errors in the cluster $C_{i}$, we still do not know the value of such a minimal error and do not have to double-check whether this minimal error satisfies an agent's specification or not. So we calculate the standard deviation of distances between historical offers and estimated offers in the cluster $C_{i}$ as follows:

$$
\sigma_{e}=\sqrt{\frac{\sum_{t_{i}, \hat{u}_{i}^{*}} e_{t_{i}, \hat{u}_{i}^{*}}^{2}}{\left|C_{i}\right|}}
$$

where $\left|C_{i}\right|$ indicates the size of cluster $C_{i}$, and $e_{t_{i}, \hat{u}_{i}^{*}}$ indicates the distance between the real offer $\left(t_{i}, \hat{u}_{i}^{*}\right)$ to the regression line $U(t)$, and $e_{t_{i}, \hat{u}_{i}^{*}}$ is calculated as follows:

$$
e_{t_{i}, \hat{u}_{i}^{*}}=\frac{a \cdot t_{i}-\hat{u}_{i}^{*}+b}{\sqrt{a^{2}+1}}
$$

If the standard deviation $\sigma_{e}$ is smaller than a predefined threshold, the regression function $U(t)$ will be considered as valid. Otherwise, the regression function $U(t)$ will be considered invalid, and the set $C_{i}$ will be divided into two subsets $C_{i}^{p}$ and $C_{i}^{q}$ as follows:

$$
\left\{\begin{array}{l}
C_{i}^{p}=\left\{\left(t_{i}, \hat{u}_{i}^{*}\right) \mid \text { if } e_{t_{i}, \hat{u}_{i}^{*}} \geqslant 0\right\} \\
C_{i}^{q}=\left\{\left(t_{i}, \hat{u}_{i}^{*}\right) \mid \text { if } e_{t_{i}, \hat{u}_{i}^{*}}<0\right\}
\end{array}\right.
$$

Then for each subsection, a linear regression function can be generated by re-employing the process introduced in this section. This procedure is repeated until the distance between all historical offers and the corresponding regression function are smaller than the predefined threshold.

\subsection{Combination of regression lines}

In the previous subsection, we introduced the approach to generate multiple regression lines in each cluster of the historical offers, so the domain area of these regression lines may be overlapped. However, 
during the negotiation, for a certain negotiation round, only one offer will be generated by an agent and is sent to opponents. So we need an approach to combine results from different regression functions together.

Let $\left\{U(t)^{i} \mid i=1 \ldots c n\right\}$ denote all linear regression lines, where $c n$ is the total number of classes. Each $U(t)^{i}$ represents one pattern in a cluster in the format as follows:

$$
U(t)^{i}=b^{i} \times t+a^{i} \quad t \in\left[t_{\text {min }}^{i}, t_{\text {max }}^{i}\right]
$$

where $t_{\text {min }}^{i}$ and $t_{\text {max }}^{i}$ defines the domain area of the function $U(t)^{i}$. Finally, the estimation of agents' behaviour in the negotiation round $t^{\prime}$ can be calculated by using Eq. (21):

$$
u_{t^{\prime}}=\operatorname{avg}\left\{\sum_{i=1}^{c n} U\left(t^{\prime}\right)^{i}, \text { if } t^{\prime} \in\left[t_{\text {min }}^{i}, t_{\text {max }}^{i}\right]\right\}
$$

\section{Probability estimation}

In the previous two sections, we proposed a power regression approach and a vector approach to predict an opponent's behaviour. However, it has to be mentioned that both of the proposed approaches can only provide an estimation on an opponent's possible behaviour, which might not exactly accord with the opponent's real behaviours. In this paper, we make an assumption that the differences $(\varepsilon)$ between the estimation behaviours and the real behaviours obey the Gaussian distribution $N\left(0, \sigma^{2}\right)$. The reason for such an assumption is because most estimated behaviours are located around the real behaviours. Thus, if the deviation $\sigma^{2}$ can be calculated, we can make a precise decision on the range of partner behaviours. It is known that there is more than $99 \%$ likelihood that an opponent's behaviours are located in the interval $[u-3 \sigma, u+3 \sigma]$. In this section, we introduce the proposed way to calculate the deviation $\sigma$ and to estimate the probability that an opponent's behaviour may happen in the future.

In order to calculate the deviation $\sigma$, we firstly calculate the distance between the estimation results $\left(u_{i}\right)$ on an opponent's offer and the real offer from historical records $\left(\hat{u}_{i}\right)$ by Eq. (22):

$$
d_{i}=\hat{u}_{i}-u_{i}
$$

It is assumed that all $d_{i}(i \in[1, n])$ obey the Gaussian distribution $N\left(0, \sigma^{2}\right)$. Then $\sigma$ can be calculated by Eq. (23):

$$
\sigma=\sqrt{\frac{\sum_{i=1}^{n}\left(d_{i}-\bar{d}\right)^{2}}{n}}
$$

where,

$$
\bar{d}=\frac{1}{n} \sum_{i=1}^{n} d_{i}
$$

Then by employing Chebyshev's inequality, we can calculate (i) the interval of an opponent's behaviour according to any accuracy requirements; and (ii) the probability that any particular behaviour may be performed by the opponent in the future. The Chebyshev inequality is given by:

$$
P(|X-\mu| \geqslant \varepsilon) \leqslant \frac{\sigma^{2}}{\varepsilon^{2}}
$$

where $X$ is an instance, $\mu$ is the mathematical expectation, $\sigma$ is the deviation, and $\varepsilon$ is the accuracy requirement.

Equation (25) indicates the probability, that the distance from a real offer $\hat{u}_{i}^{*}$ to the estimated offer $u_{i}$ is shorter than the distance $d_{i}$, and is greater than $\frac{\sigma^{2}}{\varepsilon^{2}}$. So the probability that the opponent will generate a new offer within $\left[\mu-d_{i}, \mu+d_{i}\right]$ in the future is $1-\frac{\sigma^{2}}{\varepsilon^{2}}$.

By employing Eqs. (12) and (25), agents can estimate an opponent's possible negotiation behaviours in advance, and plan a suitable strategy as a response. However, such a regression function can only illustrate the main trend of opponents' behaviours, it cannot give accurate information on how opponents change their negotiation behaviours in each negotiation round.

\section{Experiments}

In this section, we demonstrate experiments to test the proposed agent behaviour estimation approaches. We compare the proposed approaches with the Tit-ForTat approach [6] and illustrate the experimental results.

\subsection{Experimental setup}

Experiments are set up as follows. In each experiment, two agents are involved, one is the buyer and the other one is the seller. The two agents negotiate over a single issue and both of them employ the NDF 
[8] negotiation strategy. Rubinstein's alternating offers protocol [24] is employed as the negotiation protocol. The negotiation deadline is 20 rounds. Agents' utilities are normalized between 0 and 1 . During the negotiation, in order to mimic an open and dynamic negotiation environment, the seller can randomly modify its negotiation strategy, and the buyer will employ the proposed behaviour estimation approaches to predict the seller's possible behaviour in the next round. We repeat the experiment 1000 times. Both agents' initial negotiation strategies are chosen randomly. In the following subsection, we firstly illustrate the experimental results in three typical scenarios, i.e. when the seller adopts three typical negotiation strategies (conceder, linear and boulware), respectively. Then we illustrate an experimental result by allowing the seller agent to modify its negotiation strategy randomly. Finally we summarize all experimental results and give the statistical results over 1000 tests. The estimation result is evaluated by the mean and standard deviation ( $s t d)$ over the differences between the estimated offers and the real offers in all negotiation rounds. The mean and the $s t d$ are calculated as follows:

$$
d_{i}=\left|u_{i}-\hat{u}_{i}\right|
$$

where $d_{i}$ is the difference between the estimated offer $u_{i}$ and the real offer $\hat{u}_{i}$ at the $i^{t h}$ negotiation round.

$$
\text { mean }=\frac{\sum_{i=1}^{T} d_{i}}{T}
$$

and

$$
s t d=\sqrt{\frac{\sum_{i=1}^{T}\left(d_{i}-\text { mean }\right)^{2}}{T}}
$$

where $T$ is the total number of negotiation rounds.

\subsection{Scenario 1}

In the first scenario, the seller agent adopts the linear negotiation strategy. The estimation results by employing different approaches are illustrated in Fig. 6. It can be seen that the power regression line indicates the main trend of the seller's behaviour very well, but is not accurate enough to describe the details for a certain moment. The Tit-For-Tat approach indicates behaviour change at a certain moment, but has too much error. The vector analysis approach can combine the advantages of the two approaches. In Fig. 6, we

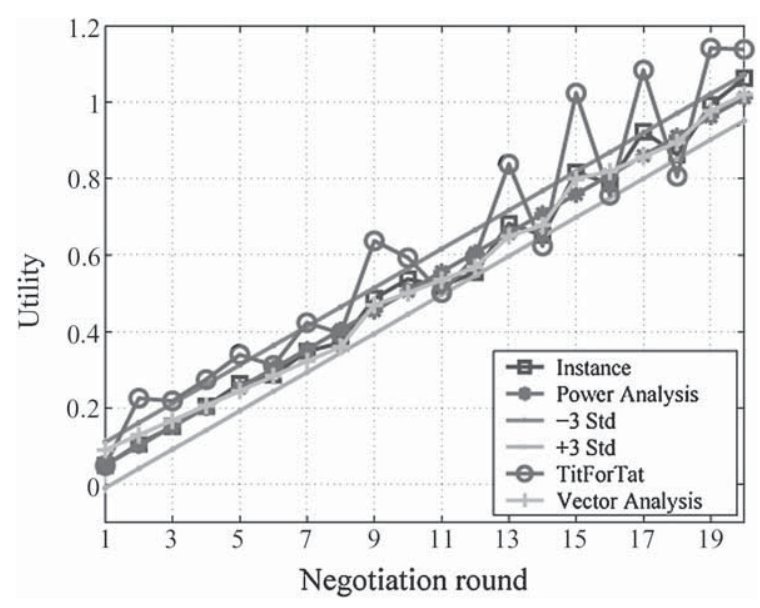

Fig. 6. Comparison of three estimation approaches when the negotiation strategy is linear.

Table 1

Estimation result of power regression when the negotiation strategy is linear

\begin{tabular}{lc}
\hline Function & Domain \\
\hline$U(t)=0.05 \times t^{1}$ & {$[1,20]$} \\
\hline
\end{tabular}

Table 2

Estimation result of vector analysis when the negotiation strategy is linear

\begin{tabular}{lll}
\hline Index & \multicolumn{1}{c}{ Function } & Domain \\
\hline 1 & $U(t)=0.039 \times t+0.009$ & {$[1,12]$} \\
2 & $U(t)=0.012 \times t+0.442$ & {$[9,14]$} \\
3 & $U(t)=0.044 \times t+0.182$ & {$[10,20]$} \\
4 & $U(t)=0.032 \times t+0.314$ & {$[16,18]$} \\
\hline
\end{tabular}

Table 3

Comparison of three estimation results when the negotiation strategy is linear

\begin{tabular}{lll}
\hline Approach & Mean & Std \\
\hline TitForTat & 0.079 & 0.057 \\
Power Regression & 0.029 & 0.02 \\
Vector Analysis & 0.024 & 0.015 \\
\hline
\end{tabular}

draw \pm 3 standard deviation lines to illustrate the $95 \%$ confidence range. Both power regression and vector analysis results are within the $95 \%$ confidence area, but some estimation results from Tit-For-Tat are out of this range. In Tables 1 and 2, we list the regression functions for both proposed approaches, respectively. The statistical evaluations on the three estimation approaches by using Eqs. (26) and (27) are listed in Table 3. The statistical results indicate that the vector analysis approach outperforms the other two approaches. 


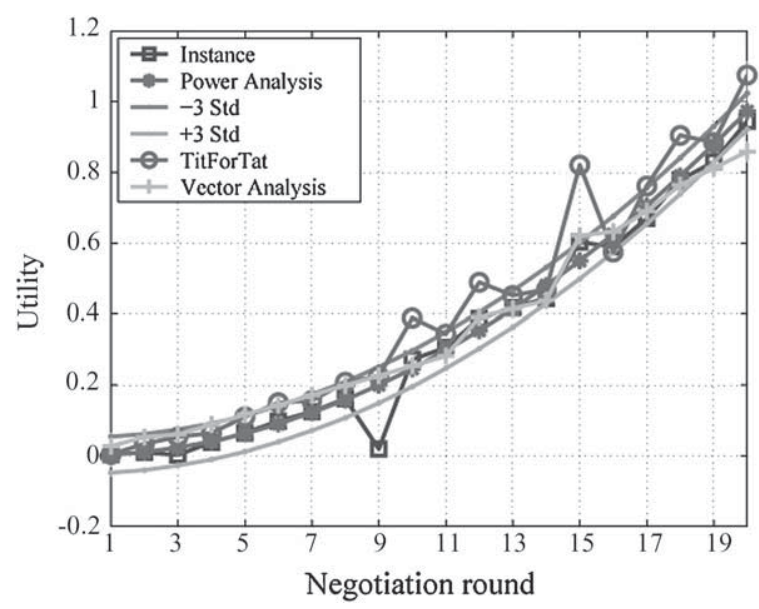

Fig. 7. Comparison of three estimation approaches when the negotiation strategy is boulware.

\subsection{Scenario 2}

In the second scenario, the seller agent employs the boulware negotiation strategy, which gives smaller concessions at the early stages of the negotiation and greater concessions at the later stages. It can be seen in Fig. 7 that the power regression function displays the main trend of the opponent's behaviour correctly. However, when the real offers from the opponent exhibit little fluctuation, the power regression function cannot identify such a change. By comparison with the power regression function, the Tit-For-Tat approach goes to the other extreme estimation result, i.e. when the real offers fluctuate, the Tit-For-Tat approach can notice such a change and give immediate responses. However, according to the experimental results, the Tit-For-Tat approach usually made a strong attempt to respond to changes in the real offers. For example, in the $10^{t h}, 12^{t h}$ and $15^{t h}$ negotiation rounds, the estimation results from the Tit-For-Tat approach are outside the \pm 3 standard deviation area. So such estimation results can be considered as invalid. The vector analysis approach can improve the estimation performance based on both the power regression approach and the Tit-For-Tat approach. According to the experimental results, not only can the vector analysis approach follow the main trend of the historical offers, but also properly indicate the fluctuations of the historical offers. Compared to the invalid estimation results generated by the Tit-For-Tat approach, the estimation results generated by the vector analysis approach in these rounds fit the historical offers very well. In Tables 4 and 5, we list the regression functions for both power regression analysis and vector analysis. Table 6
Table 4

Estimation result of power regression when the negotiation strategy is boulware

\begin{tabular}{lc}
\hline Function & Domain \\
\hline$U(t)=0.003 \times t^{1.98}$ & {$[1,20]$} \\
\hline
\end{tabular}

Table 5

Estimation result of vector analysis when the negotiation strategy is boulware

\begin{tabular}{lll}
\hline Index & Function & Domain \\
\hline 1 & $U(t)=0.025 \times t+0.027$ & {$[1,11]$} \\
2 & $U(t)=0.03 \times t-0.017$ & {$[3,10]$} \\
3 & $U(t)=0.023 \times t+0.133$ & {$[12,14]$} \\
4 & $U(t)=0.048 \times t-0.045$ & {$[15,20]$} \\
5 & $U(t)=0.068 \times t-0.422$ & {$[16,17]$} \\
\hline
\end{tabular}

Table 6

Comparison of three estimation results when the negotiation strategy is boulware

\begin{tabular}{llc}
\hline Approach & Mean & Std \\
\hline TitForTat & 0.062 & 0.051 \\
Power Regression & 0.027 & 0.027 \\
Vector Analysis & 0.03 & 0.02 \\
\hline
\end{tabular}

displays the statistical results to evaluate the accuracy of the three estimation approaches. It can be seen that, again, the vector analysis outperforms the other two approaches.

\subsection{Scenario 3}

In the third scenario, the seller agent employs the conceder negotiation strategy. According to the power regression function in Table 7 , it can be seen that the power regression analysis successfully identifies the seller agent's negotiation strategy. In Fig. 8, it can be seen that the estimation results generated by the vector analysis approach fit the historical offers properly, except in the $16^{\text {th }}$ negotiation round. The regression functions for vector analysis are listed in Table 8. The Tit-For-Tat approach does not perform well in this experiment. Almost $50 \%$ of the estimation results do not fit the historical offers, and five (25\%) estimation results even reside outside the \pm 3 standard deviation area. The statistic evaluations on the three estimation approaches are listed in Table 9. It can be seen that the power regression approach achieves a similar result as the vector analysis approach, but the Tit-For-Tat approach does not perform well. 


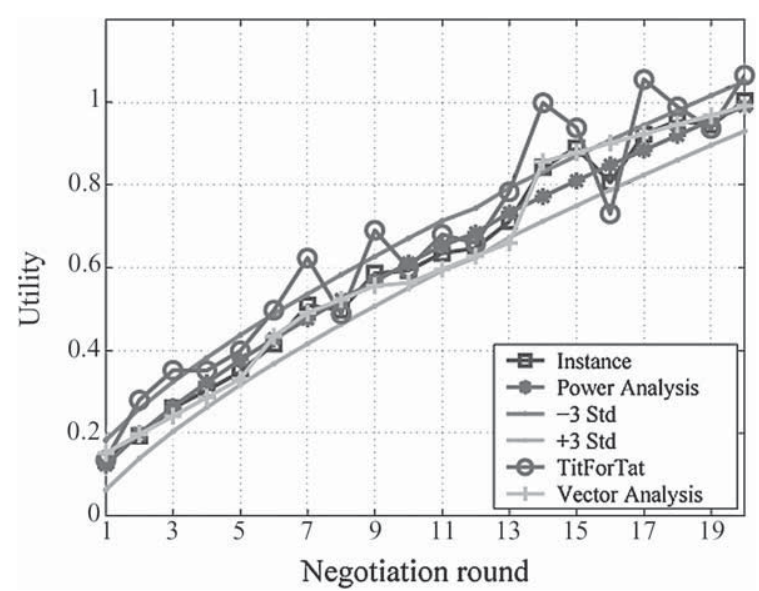

Fig. 8. Comparison of three estimation approaches when the negotiation strategy is conceder.

Table 7

Estimation result of power regression when the negotiation strategy is conceder

\begin{tabular}{lc}
\hline Function & Domain \\
\hline$U(t)=0.123 \times t^{0.7}$ & {$[1,20]$} \\
\hline
\end{tabular}

Table 8

Estimation result of vector analysis when the negotiation strategy is conceder

\begin{tabular}{lll}
\hline Index & \multicolumn{1}{c}{ Function } & Domain \\
\hline 1 & $U(t)=0.045 \times t+0.152$ & {$[1,5]$} \\
2 & $U(t)=0.032 \times t+0.273$ & {$[6,13]$} \\
3 & $U(t)=0.032 \times t+0.32$ & {$[7,9]$} \\
4 & $U(t)=0.022 \times t+0.562$ & {$[14,20]$} \\
\hline
\end{tabular}

Table 9

Comparison of three estimation results when the negotiation strategy is conceder

\begin{tabular}{llc}
\hline Approach & Mean & Std \\
\hline TitForTat & 0.06 & 0.04 \\
Power Regression & 0.027 & 0.02 \\
Vector Analysis & 0.023 & 0.02 \\
\hline
\end{tabular}

\subsection{Scenario 4}

In the fourth scenario, the seller agent is allowed to modify its negotiation strategy randomly during the negotiation. The experimental results are illustrated in Fig. 9. It can be seen that the seller agent's offer fluctuates greatly and frequently. For the estimation results generated by employing the Tit-For-Tat approach, $25 \%$ of estimation results are located out of the $\pm 3 \sigma$ confidence area. As displayed in Table 12, the average er-

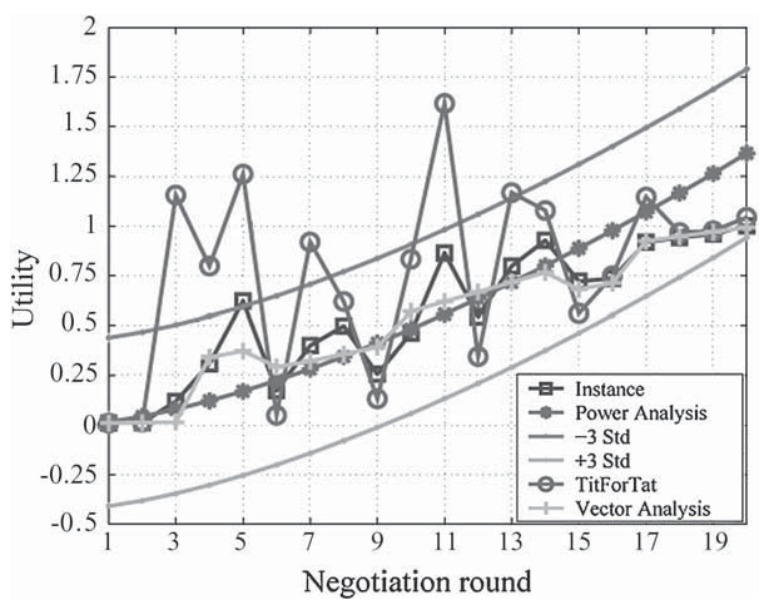

Fig. 9. Comparison of three estimation approaches when the negotiation strategy is dynamically modified.

Table 10

Estimation result of power regression when the negotiation strategy is dynamically modified

\begin{tabular}{lc}
\hline Function & Domain \\
\hline$U(t)=0.152 \times t^{1.5}$ & {$[1,20]$} \\
\hline
\end{tabular}

Table 11

Estimation result of vector analysis when the negotiation strategy is dynamically modified

\begin{tabular}{lll}
\hline Index & \multicolumn{1}{c}{ Function } & Domain \\
\hline 1 & $U(t)=0.002 \times t+0.01$ & {$[1,3]$} \\
2 & $U(t)=0.032 \times t+0.246$ & {$[4,15]$} \\
3 & $U(t)=0.023 \times t+0.066$ & {$[6,9]$} \\
4 & $U(t)=0.063 \times t+0.064$ & {$[7,14]$} \\
5 & $U(t)=0.038 \times t+0.14$ & {$[10,16]$} \\
6 & $U(t)=0.023 \times t+0.557$ & {$[17,20]$} \\
\hline
\end{tabular}

ror of Tit-For-Tat in this case is almost 0.4 , and the standard deviation is around 0.57 . These values indicate that the estimated results generated by the Tit-ForTat approach are very unstable and inaccurate when agents modify their negotiation strategy randomly. By Comparison, the power regression approach generates a better result. The power regression function is listed in Table 10. According to Table 12, because the standard deviation of errors in estimated results is around 0.14 , so almost all results are located inside the $\pm 2 \sigma$ confident area. Again, the vector approach provides best estimation results among them in this case. It can be seen that the regression lines generated by the vector method follow the real instances much better than the other approaches. Nearly $50 \%$ of estimated offer are located on the real offers. As shown in Table 12, 
Table 12

Comparison of three estimation results when the negotiation strategy is dynamically modified

\begin{tabular}{llc}
\hline Approach & Mean & Std \\
\hline TitForTat & 0.392 & 0.578 \\
Power Regression & 0.181 & 0.141 \\
Vector Analysis & 0.104 & 0.102 \\
\hline
\end{tabular}

Table 13

Comparison of three estimation results by considering three negotiation strategies

\begin{tabular}{lll}
\hline Approach & Mean & Std \\
\hline TitForTat & 0.551 & 2.138 \\
Power Regression & 0.097 & 0.067 \\
Vector Analysis & 0.074 & 0.05 \\
\hline
\end{tabular}

both the average error and the standard deviation of the vector approach are only around 0.1 .

\subsection{Summary}

In order to have a comprehensive comparison between these three estimation approaches, we repeat the experiment 1000 times. The seller agent is allowed to adopt different negotiation strategies randomly. The statistical evaluation results over the 1000 experiments are displayed in Table 13. It can be seen that on average, the vector analysis approach achieves the best estimation performance, which has 0.074 error in average and 0.05 in the standard deviation of errors. The result from the power regression approach is also acceptable, which is 0.097 error in average and 0.067 in the standard deviation of errors. However, the performance of the Tit-For-Tat approach is not satisfied. The average error of the Tit-For-Tat approach is almost eight times greater than the vector approach. In summary, it is found that the vector analysis approach can provide the best results in an opponent's behaviour prediction.

\section{Related work}

In this section, we introduce some related works. In [22], we proposed a regression-based model to estimate negotiation opponents' behaviour. However, the regression-based approach is good at indicating opponents' main trend on negotiation strategy, but may not sensitive enough to catch opponents' changes on bidding in each negotiation round. Therefore, we introduce a vector-based estimation approach as a supplement of regression-based model in this paper.
In [25], Schapire et al. proposed a machine learning approach based on a boosting algorithm. Initially, the estimation problem is reduced to a classification problem. All training data are arranged in ascending order and then partitioned into groups equally. For each of the breakpoints, a learning algorithm is employed to estimate the probability that a new bid at least should be greater than the breakpoint. The final result of this learning approach is a function which gives minimal error rate between the estimated bid and the real one. Based on this function, agent behaviours can be estimated. However, the accuracy of this approach is limited by the training data and classification approach. So applications based on this approach can hardly achieve a satisfactory level when negotiations happen in an open and dynamic environment.

In [10], Gal and Pfeffer presented another machine learning approach based on a statistical method. The proposed approach is firstly trained by agent behaviours according to their types. Then for an unknown agent, it will be classified into a known kind of agent according to their similarities. Finally, based on these probabilities, the unknown agent behaviour is estimated by combining all known agent behaviours. The limitation of this approach is that, in reality, it is impossible to train a system with all different types of agents. Therefore if an unknown agent belongs to a type which is excluded from the system, the estimation result may not reach an acceptable accuracy level.

Chajewska et al. [3] proposed a decision-tree approach to learn and estimate an agent's utility function. The authors assumed that each agent is rational and looks for maximum expected utility in negotiation. Firstly, a decision tree is established which contains all possible endings for the negotiation. Each possible ending is assigned a particular utility value and possibility. Based on the partner's previous decisions on the decision tree, a linear function can be generated analogous to the partner's utility function, and each item in the function comes from an internal node in the decision tree. The limitation of this approach is the requirement that all possible negotiation endings and the corresponding probabilities should be estimated in advance, which is impossible in some application domains when the variance of negotiation issues is discrete or the negotiation environment is open and dynamic.

Brzostowski and Kowalczyk [2] presented a way to estimate partners' behaviours based only on the historical offers in the current negotiation. In the first place, partner types are estimated based on the given 
functions. For each type of agent, a distinct prediction function is given to estimate agent behaviours. Therefore, based on the classification of partner types and their individual estimation functions, the proposed approach can predict partner behaviours in the next negotiation cycle. However, a partner can only perform as a time-dependent agent or a behaviour-dependent agent, which limits some applications. Also the accuracy of classification on partner types may impact the accuracy of prediction results.

Hindriks and Tykhonov [15] proposed a generic framework based on a Bayesian model to learn opponent negotiation behaviour in multiple issue negotiation. The purpose of this framework is to learn both the opponent's negotiation preference and the utility function. The opponent's preference is estimated based on an assumption that the opponent will make a greater concession on a less-valued issue and a smaller concession on a more-valued issue. Through comparing the opponent's concessions for each issue, the opponent's preference can be estimated. In order to learn the opponent's utility function, three basic functions are proposed. The opponent's utility function is finally represented as a combination of the three possible functions. By using the estimated preference and utility function, an agent can efficiently search for an optimal negotiation outcome. However, this approach can only generate a satisfying result when the opponent performs a relatively simple behaviour. If the opponent's behaviour becomes complex and changeful, it will not be estimated easily and an optimal negotiation outcome may not be reached effectively.

Maheswaran et al. [17] proposed a Criticality-Sensitive Coordination (CSC) system to handle coordination problems in complex environments. In a dynamic, uncertain and nonlinear environment, agents may only have partial knowledge of the team reward function, and cannot accurately choose the optimal policy to maximize the global reward. In order to avoid harming the current policy when an agent changes its policy, a Predictability and Criticality Metrics (PCM) is proposed, where the predictability limits the policy modification within an acceptable area, and criticality metrics evaluate a potential policy modification by considering the nonlinear effect. By combining these two processes, even though it cannot guarantee that a global maximal team reward can be reached, it can avoid great damage on the team reward. This approach can be applied on a coordination system where stability is the primary consideration. However, since this approach does not pay attention to searching for the optimal policy, the performance of this approach may not be conspicuous by considering the global team reward.

In [9], Fatima et al. studied bilateral multi-issue negotiation between self-interested agents whose utility functions are nonlinear. The authors argued that even though the package deal procedure leads multiple negotiation to Pareto optimality, computing the equilibrium for the package deal procedure is not always easy, especially for non-linear utility functions. In order solve such a problem, the authors introduced two approaches: (1) to approximate non-linear utility functions by linear functions; and (2) to use the simultaneous procedure to negotiate issues in parallel but independently. By employing these two approaches, approximate equilibrium will be found in polynomial time. This paper also showed that although the package deal procedure is known to generate Pareto optimal outcomes, the simultaneous procedure may outperform in some cases by considering economic properties. However, the first approach may fail to reach an optimal outcome when an approximate line is hard to find, and the second approach may fail to reach an optimal outcome when the negotiated issues are not absolutely independent.

In [26], Schvartzman and Wellman proposed a general approach to automatically search for equilibrium strategies in negotiation through reinforcement learning, and applied this methodology to continuous double auction game. During the game, a new bidding strategy will be generated by using reinforcement learning, and tested by negotiators. If the new proposed bidding strategy can improve the reinforcement learning model, then it will be accepted as a potential bidding strategy, otherwise, it will be discarded by the agent. The process will complete when no further improvement can be made on the reinforcement learning model. The major difference between this paper and our work is that we pay more attention to estimate opponents' bidding behaviours and to modify agents' existing negotiation strategy, but not focus on generating different bidding strategies.

By comparing our approach with the above opponent behaviour estimation approaches, our proposed approach has two attractive merits. (1) The proposed approach does not need any training or preparation in advance, and it can estimate an opponent's behaviour based only on the current historical records and generate reasonable estimation results in a timely manner. So, an agent can save its time, and increase the negotiation efficiency by employing the proposed approach; 
and (2) the proposed approach estimates an opponent's behaviour according to the probability that each particular behaviour will happen in the future. So an agent can adopt the estimation results as well as the corresponding probabilities to guide its own negotiation behaviours efficiently in the future.

\section{Conclusion and future work}

In this paper, we proposed a regression approach and a vector approach to estimate an opponent's negotiation behaviours. The regression approach is good at indicating the main trend of an opponent's negotiation behaviour, and the vector approach is sensitive to changes on an opponent's behaviour. The proposed approaches do not require any prior training process, and can also indicate the likelihood of each estimated result. The experimental results indicate the efficacy and efficiency of the proposed approach by comparison with the Tit-For-Tat approach.

Future work on this research will focus on two directions. (1) To extend the proposed approach from single-issue to multi-issue negotiation, and (2) to extend the proposed approach from bilateral to multilateral negotiation.

\section{References}

[1] J. Brzostowski and R. Kowalczyk, On possibilistic case-based reasoning for selecting partners in multi-agent negotiation, in: Proc. of 17th Aus. Conf. on Artificial Intelligence, Vol. 3339, 2004, pp. 694-705.

[2] J. Brzostowski and R. Kowalczyk, Predicting partner's behaviour in agent negotiation, in: Proc. of 5th Int. Conf. on Autonomous Agents and Multiagent Systems (AAMASO6), 2006, pp. $355-361$.

[3] U. Chajewska, D. Koller, and D. Ormoneit, Learning an agent's utility function by observing behavior, in: Proc. of 18th Int. Conf. on Machine Learning (ICML01), 2001, pp. 35-42.

[4] A. Chavez, D. Dreilinger, R. Guttman, and P. Maes, A real-life experiment in creating an agent marketplace, Lecture Notes in Computer Science 1198(2) (2001), 160-179.

[5] A. Chavez and P. Maes, Kabash: An agent marketplace for buying and selling goods, in: Proc. of 1st Int. Conf. on the Practical Application of Intelligent Agents and Multi-Agent Technology (PAAM'96), 1996, pp. 75-90.

[6] P. Faratin, C. Sierra, and N. Jennings, Negotiation decision functions for autonomous agents, Journal of Robotics and Autonomous Systems 24(3-4) (1998), 159-182.

[7] S. Fatima, M. Wooldridge, and N. Jennings, Optimal agendas for multi-issue negotiation, in: Proc. of 2nd Int. Conf. on Autonomous Agents and Multi-Agent Systems (AAMASO3), ACM Press, 2003, pp. 129-136.
[8] S. Fatima, M. Wooldridge, and N. Jennings, An agenda-based framework for multi-issue negotiation, Artificial Intelligence 152(1) (2004), 1-45.

[9] S. Fatima, M. Wooldridge, and N. Jennings, An analysis of feasible solutions for multi-issue negotiation involving nonlinear utility functions, in: Proc. of 8th Int. Conf. on Autonomous Agents and Multiagent Systems (AAMAS09), 2009, pp. 10411048.

[10] Y. Gal and A. Pfeffer, Predicting people's bidding behavior in negotiation, in: Proc. of 5th Int. Conf. on Autonomous Agents and Multiagent Systems (AAMAS06), 2006, pp. 370-376.

[11] A. Gupta, B. Su, and Z. Walter, Risk profile and consumer shopping behavior in electronic and traditional channels, Decision Support Systems 38(3) (2004), 347-367.

[12] R. Guttman and P. Maes, Cooperative vs. competitive multiagent negotiations in retail electronic commerce, in: Proc. of 2nd Int. Workshop on Cooperative Information Agents (CIA'98), 1998, pp. 135-141.

[13] R. Guttman and P. Maes, Agent-mediated integrative negotiation for retail electronic commerce, Lecture Notes in Computer Science 1571(2) (1999), 70-90.

[14] M. He, N. Jennings, and H. Leung, On agent-mediated electronic commerce, IEEE Transaction on Knowledge and Data Engineering 15(4) (2003), 985-1003.

[15] K. Hindriks and D. Tykhonov, Opponent modelling in automated multi-issue negotiation using bayesian learning, in: Proc. of 7th Int. Conf. on Autonomous Agents and Multiagent Systems (AAMAS08), 2008, pp. 331-338.

[16] S. Kraus, Strategic Negotiation in Multiagent Environments, The MIT Press, Cambridge, Massachusetts, 2001.

[17] R. Maheswaran, P. Szekely, M. Becker, S. Fitzpatrick, G. Gati, J. Jin, R. Neches, N. Noori, C. Rogers, and R. Sanchez, Predictability \& criticality metrics for coordination in complex environments, in: Proc. of 7 th Int. Conf. on Autonomous Agents and Multiagent Systems (AAMAS08), 2008, pp. 647654.

[18] S. Munroe, M. Luck, and M. d'Inverno, Motivation-based selection of negotiation partners. in: Proc. of 3rd Int. Conf. on Autonomous Agents and Multiagent Systems (AAMAS04), 2004, pp. $1520-1521$

[19] I. Papaioannou, I. Roussaki, and M. Anagnostou, Neural networks against genetic algorithms for negotiating agent behaviour prediction, Web Intelligence and Agent Systems 6(2) (2008), 217-233.

[20] S. Parsons, C. Sierra, and N. Jennings, Agents that reason and negotiate by arguing, Journal of Logic and Computation 8(3) (1998), 261-292.

[21] F. Ren, K. Sim, and M. Zhang, Market-driven agents with uncertain and dynamic outside options, in: Proc. of 6th Int. Conf. on Autonomous Agents and Multi-Agent Systems (AAMAS07), 2007, pp. 721-723.

[22] F. Ren and M. Zhang, Prediction partners' behaviors in negotiation by using regression analysis, in: Proc. of 2 nd Int. Conf. on Knowledge Science, Engineering and Management (KSEM07), 2007, pp. 165-176.

[23] J. Rodríguez-Aguilar, F. Martín, P. Noriega, P. Garcia, and C. Sierra, Toward a testbed for trading agents in electronic auction markets, AI Commun.: Eur. Journal Artificial Intelligence 11(1) (1998), 5-19.

[24] A. Rubinstein, Perfect equilibrium in a bargaining model, Econometrica 50(1) (1982), 97-109. 
[25] R. Schapire, P. Stone, D. McAllester, M. Littman, and J. Csirik, Modeling auction price uncertainty using boosting-based conditional density estimation, in: Proc. of 19th Int. Conf. on Machine Learning (ICML02), 2002, pp. 546-553.

[26] L. Schvartzman and M. Wellman, Stronger CDA strategies through empirical game-theoretic analysis and reinforcement learning, in: Proc. of the 8th Int. Conf. on Autonomous Agents and Multiagent Systems (AAMAS09), 2009, pp. 249-256.

[27] K. Sim, Negotiation agents that make prudent compromises and are slightly flexible in reaching consensus, Computational Intelligence, Special issue on Business Agents and the Semantic Web, pp. 643-662, 2004.
[28] K. Sim, Equilibria, prudent compromises, and the "waiting" game, IEEE Transaction on Systems, Man and Cybernetics, Part B: Cybernetics 35(4) (2005), 712-724.

[29] K. Sim and C. Choi, Agents that react to changing market situations, IEEE Transaction on Systems, Man and Cybernetics, Part B: Cybernetics 33(2) (2003), 188-201.

[30] P. Wurman, M. Wellman, and W. Walsh, The Michigan internet auctionBot: A configurable auction server for human and software agents, in: Proc. of 2nd Int. Conf. Autonomous Agents, 1998, pp. 301-308.

[31] D. Zeng and K. Sycara, Bayesian learning in negotiation, Int. Journal of Human-Computer Studies 48(1) (1998), 125-141. 
Copyright of Web Intelligence \& Agent Systems is the property of IOS Press and its content may not be copied or emailed to multiple sites or posted to a listserv without the copyright holder's express written permission. However, users may print, download, or email articles for individual use. 